\title{
Existence of solutions for nonlinear Caputo-Hadamard fractional differential equations via the method of upper and lower solutions
}

\author{
Yunru Baia,b, Hua Kong ${ }^{\mathrm{b}, *}$ \\ a Institute of Computer Science, Faculty of Mathematics and Computer Science, Jagiellonian University, ul. Lojasiewicza 6, 30-348 \\ Krakow, Poland. \\ ${ }^{b}$ Data Recovery Key Laboratory of Sichuan Province, College of Mathematics and Information Science, Neijiang Normal University, \\ Neijiang 641100, China.
}

Communicated by X.-J. Yang

\begin{abstract}
The purpose of this paper is devoted to consider the existence of solutions for a class of nonlinear Caputo-Hadamard fractional differential equations with integral terms ((CHFDE), for short). Firstly, by applying the semi-group property of Hadamard fractional integral operator, a necessary condition of solvability for (CHFDE) is established. Then, under the suitable conditions, we prove the solution set of (CHFDE) is nonempty by using the method of upper and lower solutions, and ArzelàAscoli theorem. Finally, we present several numerical examples to explicate the main results. (C)2017 All rights reserved.
\end{abstract}

Keywords: Caputo-Hadamard derivative, fractional differential equations, upper and lower solutions, monotone sequences, Arzelà-Ascoli theorem.

2010 MSC: 49J53, 49K40, 90C33, 90C46.

\section{Introduction}

The fractional calculus ((FC), for short) deals with extensions of derivatives and integrals to noninteger orders, which was started to be considered deeply as a powerful tool to reveal the hidden aspects of the dynamics of the complex or hyper complex systems (see $[1,4,8,10,14,15,17,18,24-26]$ ). In the last thirty years or so, the theory of (FC) has produced an abundance of important results both in pure and applied mathematics as well as in other fields as for example: physics, chemistry, biology, economics, control theory, signal and image processing, biophysics, blood flow phenomena, aerodynamics, fitting of experimental data, etc. (see [11-13, 22, 28-31]), as it is allowed mathematical formulations for new classes of interesting problems (see $[3,5,6,19,20,23,32]$ ).

To our knowledge, the fractional integral and derivative operators are usually studied in the sense of Riemann-Liouville, Caputo or Grünwald-Letnikov. However, Hadamard fractional derivative operator was introduced and studied in order to consider the problems, which is different completely to the ones in the sense of Riemann-Liouville, Caputo and Grünwald-Letnikov (see [2, 7, 9, 10, 16, 21]). In the present

\footnotetext{
*Corresponding author

Email addresses: yunrubai@163.com (Yunru Bai), konghua2008@126.com (Hua Kong)
} 
paper, we shall consider the following fractional nonlinear differential system involving Hadamard fractional integral term

$$
\left\{\begin{array}{l}
{ }^{C} D_{a^{+}}^{\alpha} x(t)=f\left(t, x(t), I_{a^{+}}^{\alpha} x(t)\right), t \in[a, b], \\
x(a)=x_{a},
\end{array}\right.
$$

where ${ }^{C} D_{a^{+}}^{\alpha}$ and $I_{a^{+}}^{\alpha}$ stand for the Caputo-Hadamard derivative and Hadamard integral operators (see Definitions 2.1 and 2.4, below), respectively, $f:[a, b] \times \mathbb{R} \times \mathbb{R} \rightarrow \mathbb{R}, x_{a} \in \mathbb{R}$, and $0<a<b<\infty$.

The rest of paper is structured as follows. Section 2 contains basic definitions and results needed in the sequel. Section 3 is devoted to present the main results describing the existence of solutions for Caputo-Hadamard fractional differential equation (1.1). In Section 4, we show several numerical examples to explicate our results.

\section{Preliminaries}

In this section we collect a few notions and results to be used later in the paper. In the sequel, we denote $A C(a, b ; \mathbb{R})$ the function space, by $\mathbb{R}$-valued absolutely continuous functions on $[a, b]$. In the begin, we recall fractional operators in the sense of Hadamard.

Definition $2.1([7,10])$. Let $0<a<b<\infty$ and $x:[a, b] \rightarrow \mathbb{R}$. The Hadamard fractional integral of order $\alpha>0$ of $x$ is defined by

$$
\mathrm{I}_{\mathrm{a}^{+}}^{\alpha} \chi(\mathrm{t})=\frac{1}{\Gamma(\alpha)} \int_{\mathrm{a}}^{\mathrm{t}}\left(\ln \frac{\mathrm{t}}{\mathrm{s}}\right)^{\alpha-1} \frac{\chi(\mathrm{s})}{\mathrm{s}} \mathrm{ds} \text { for } \mathrm{t} \in[\mathrm{a}, \mathrm{b}],
$$

where $\Gamma$ stands for the well-known Gamma function by

$$
\Gamma(\alpha)=\int_{0}^{\infty} \mathrm{t}^{\alpha-1} e^{-\mathrm{t}} \mathrm{dt}
$$

Definition 2.2 ([7, 10]). Let $0<a<b<\infty$ and $x:[a, b] \rightarrow \mathbb{R}$. The Hadamard fractional derivative of order $\alpha \in(0,1]$ of $x$ is defined by

$$
D_{a^{+}}^{\alpha} x(t)=\frac{1}{\Gamma(1-\alpha)} t \frac{d}{d t} \int_{a}^{t}\left(\ln \frac{t}{s}\right)^{-\alpha} \frac{x(s)}{s} d s \text { for } t \in[a, b] .
$$

Obviously, we can obtain

$$
I_{a^{+}}^{\alpha}\left(\ln \frac{t}{a}\right)^{\beta-1}=\frac{\Gamma(\beta)}{\Gamma(\beta+\alpha)}\left(\ln \frac{t}{a}\right)^{\beta+\alpha-1}, \quad D_{a^{+}}^{\alpha}\left(\ln \frac{t}{a}\right)^{\beta-1}=\frac{\Gamma(\beta)}{\Gamma(\beta-\alpha)}\left(\ln \frac{t}{a}\right)^{\beta-\alpha-1}
$$

for $t \in[a, b]$.

Now, we review some significant properties for fractional Hadamard integral and derivative operators in which their proof can be found in $[7,10]$.

Lemma 2.3 ([7,10]). Let $\alpha, \beta$ be such that $\alpha>0$ and $\beta>0$.

(i) If $1 \leqslant p<\infty$, then for $x \in \mathrm{L}^{\mathrm{p}}(\mathrm{a}, \mathrm{b} ; \mathbb{R})$, we have

$$
\mathrm{I}_{\mathrm{a}^{+}}^{\beta} \mathrm{I}_{\mathrm{a}^{+}}^{\alpha} \chi(\mathrm{t})=\mathrm{I}_{\mathrm{a}^{+}}^{\alpha+\beta} \chi(\mathrm{t}) \text { for } \mathrm{t} \in[\mathrm{a}, \mathrm{b}] .
$$

(ii) If $1 \leqslant p<\infty$ and $\alpha>\beta$, then for $x \in \mathrm{L}^{\mathrm{p}}(\mathrm{a}, \mathrm{b} ; \mathbb{R})$, we have

$$
\mathrm{D}_{\mathrm{a}^{+}}^{\beta} \mathrm{I}_{\mathrm{a}^{+}}^{\alpha} \chi(\mathrm{t})=\mathrm{I}_{\mathrm{a}^{+}}^{\alpha-\beta} \chi(\mathrm{t}) \text { for } \mathrm{t} \in[\mathrm{a}, \mathrm{b}] .
$$

Definition 2.4. Let $0<a<b<\infty$ and $x:[a, b] \rightarrow \mathbb{R}$. The Caputo-Hadamard fractional derivative of order $\alpha \in(0,1]$ of $x$ is defined by

$$
{ }^{C} D_{a^{+}}^{\alpha} x(t)=D_{a^{+}}^{\alpha}[x(t)-x(a)] \text { for } t \in[a, b] .
$$


Remark 2.5. It is obvious that if $x \in A C(a, b ; \mathbb{R})$, then Caputo-Hadamard fractional derivative, Definition 2.4, has the following equivalent formulation

$$
{ }^{c} D_{a^{+}}^{\alpha} \chi(t)=\frac{1}{\Gamma(1-\alpha)} \int_{a}^{t}\left(\ln \frac{t}{s}\right)^{-\alpha} \chi^{\prime}(s) d s \text { for } t \in[a, b] .
$$

We conclude this section by recalling the following component properties for Caputo-Hadamard fractional operators.

Lemma $2.6([7,10])$. Let $\alpha>0$ be such that $n=[\alpha]+1$.

(i) If $x \in \mathrm{C}(\mathrm{a}, \mathrm{b} ; \mathbb{R})$, then

$$
{ }^{C} D_{a^{+}}^{\alpha}\left(I_{a^{+}}^{\alpha} \chi(t)\right)=x(t) \text { for } t \in[a, b]
$$

(ii) If $x \in A C(a, b ; \mathbb{R})$, then

$$
\mathrm{I}_{\mathrm{a}^{+}}^{\alpha}\left({ }^{\mathrm{C}} \mathrm{D}_{\mathrm{a}^{+}}^{\alpha} x(\mathrm{t})\right)=x(\mathrm{t})-x(\mathrm{a}) \text { for } \mathrm{t} \in[\mathrm{a}, \mathrm{b}]
$$

\section{Main results}

In this section, we focus our attention on the existence of solutions for fractional nonlinear differential system (1.1).

Theorem 3.1. Let $\mathrm{f}:[\mathrm{a}, \mathrm{b}] \times \mathbb{R} \times \mathbb{R} \rightarrow \mathbb{R}$ be a continuous function. Assume that $\mathrm{x} \in \mathrm{C}(\mathrm{a}, \mathrm{b} ; \mathbb{R})$ is a solution of the following integral equation

$$
x(t)=x_{a}+\frac{1}{\Gamma(\alpha)} \int_{a}^{t}\left(\ln \frac{t}{s}\right)^{\alpha-1} \frac{f\left(s, x(s), I_{a^{+}}^{\alpha} x(s)\right)}{s} \text { ds for } t \in[a, b],
$$

then it also resolves the fractional nonlinear differential equation (1.1).

Proof. Assume that $x \in C(a, b ; \mathbb{R})$ is a solution of the integral equation (3.1). Obviously, we obtain $x(a)=$ $x_{a}$ and $t \mapsto I_{a^{+}}^{\alpha} x(t) \in C(a, b ; \mathbb{R})$. The continuity of $f$ and definition of Hadamard integral $I_{a^{+}}^{\alpha}$ guarantee that $t \mapsto f\left(t, x(t), I_{a^{+}}^{\alpha} \chi(t)\right)$ is continuous as well and

$$
\left.\mathrm{I}_{\mathrm{a}^{+}}^{\alpha} \mathrm{f}\left(\mathrm{t}, x(\mathrm{t}), \mathrm{I}_{\mathrm{a}^{+}}^{\alpha} x(\mathrm{t})\right)\right|_{\mathrm{t}=\mathrm{a}}=0 .
$$

Since, $t \mapsto I_{a^{+}}^{\alpha} f\left(t, x(t), I_{a^{+}}^{\alpha} x(t)\right)$ is continuous, then we have $x$ is differential for a.e. $t \in(a, b)$, see (3.1), i.e., $x \in A C(a, b ; \mathbb{R})$. From Lemma 2.6, we have

$$
{ }^{C} D_{a^{+}}^{\alpha} I_{a^{+}}^{\alpha} f\left(t, x(t), I_{a^{+}}^{\alpha} x(t)\right)=f\left(t, x(t), I_{a^{+}}^{\alpha} x(t)\right) \text { for } t \in[a, b] .
$$

On the other hand, Remark 2.5 reveals

$$
{ }^{c} D_{a^{+}}^{\alpha}\left[x(t)-x_{a}\right]=\frac{1}{\Gamma(1-\alpha)} \int_{a}^{t}\left(\ln \frac{t}{s}\right)^{-\alpha}\left[x(s)-x_{a}\right]^{\prime} d s=\frac{1}{\Gamma(1-\alpha)} \int_{a}^{t}\left(\ln \frac{t}{s}\right)^{-\alpha} x^{\prime}(s) d s={ }^{c} D_{a^{+}}^{\alpha} x(t)
$$

for $t \in[a, b]$.

By all above, we conclude that $x \in C(a, b ; \mathbb{R})$ is a solution of fractional nonlinear differential equation (1.1), which completes the proof of the theorem.

Now, we introduce the concept of upper and lower solutions for integral equation (3.1), which plays a remarkable role in our work.

Definition 3.2. Let $(\underline{x}, \bar{x}) \in C(a, b ; \mathbb{R}) \times C(a, b ; \mathbb{R})$. A pair of functions $(\underline{x}, \bar{x})$ is called to be upper and lower solutions of fractional integral equation (3.1), respectively, if

$$
\underline{x}(t) \leqslant x_{a}+\frac{1}{\Gamma(\alpha)} \int_{a}^{t}\left(\ln \frac{t}{s}\right)^{\alpha-1} \frac{f\left(s, \underline{x}(s), I_{a^{+}}^{\alpha} \underline{x}(s)\right)}{s} \text { ds for all } t \in[a, b],
$$


and

$$
\bar{x}(t) \geqslant x_{a}+\frac{1}{\Gamma(\alpha)} \int_{a}^{t}\left(\ln \frac{t}{s}\right)^{\alpha-1} \frac{f\left(s, \bar{x}(s), I_{a^{+}}^{\alpha} \bar{x}(s)\right)}{s} \text { ds for all } t \in[a, b] .
$$

Let $(\underline{x}, \bar{x})$ be a pair of upper and lower solutions of fractional integral equation (3.1). In the sequel, we denote an admissible set of solutions for fractional integral equation (3.1) governed by a pair of upper and lower solutions $(\underline{x}, \bar{x})$ as follows

$$
\mathrm{U}_{(\underline{x}, \bar{x})}:=\{x \in C(a, b ; \mathbb{R}): \underline{x}(t) \leqslant x(t) \leqslant \bar{x}(t), t \in[a, b] \text { and } x \text { is a solution of }(3.1)\} .
$$

Theorem 3.3. Let $\mathrm{f} \in \mathrm{C}\left([\mathrm{a}, \mathrm{b}] \times \mathbb{R}^{2} ; \mathbb{R}\right)$. Assume that $(\underline{\mathrm{x}}, \overline{\mathrm{x}}) \in \mathrm{C}(\mathrm{a}, \mathrm{b} ; \mathbb{R}) \times \mathrm{C}(\mathrm{a}, \mathrm{b} ; \mathbb{R})$ is a pair of upper and lower solutions of fractional integral equation (3.1) with $\underline{x}(t) \leqslant \bar{x}(t)$ for $t \in[a, b]$. If $(x, y) \mapsto f(t, x, y)$ is nondecreasing, that is

$$
f\left(t, x_{1}, y_{1}\right) \leqslant f\left(t, x_{2}, y_{2}\right) \text { for } x_{1} \leqslant x_{2} \text { and } y_{1} \leqslant y_{2} \text {, }
$$

then there exist maximal and minimal solutions $\mathrm{x}_{\mathrm{M}}, \mathrm{x}_{\mathrm{L}} \in \mathrm{U}_{(\underline{x}, \overline{\mathrm{x}})}$ in $\mathrm{U}_{(\underline{x}, \overline{\mathrm{x}})}$, i.e., for each $\mathrm{x} \in \mathrm{U}_{(\underline{x}, \bar{x})}$ one has

$$
x_{L}(t) \leqslant x(t) \leqslant x_{M}(t) \text { for } t \in[0, T] .
$$

Proof. To this end, we construct two sequences $\left\{y_{n}\right\}$ and $\left\{z_{n}\right\}$ as follows

$$
\left\{\begin{array}{l}
y_{0}=\underline{x}, \\
y_{n+1}(t)=x_{a}+\frac{1}{\Gamma(\alpha)} \int_{a}^{t}\left(\ln \frac{t}{s}\right)^{\alpha-1} \frac{f\left(s, y_{n}(s), I_{a^{+}}^{\alpha} y_{n}(s)\right)}{s} d s, t \in[a, b] \text { and } n=0,1, \ldots,
\end{array}\right.
$$

and

$$
\left\{\begin{array}{l}
z_{0}=\bar{x} \\
z_{n+1}(t)=x_{a}+\frac{1}{\Gamma(\alpha)} \int_{a}^{t}\left(\ln \frac{t}{s}\right)^{\alpha-1} \frac{f\left(s, z_{\mathfrak{n}}(s), I_{a^{+}}^{\alpha} z_{\mathfrak{n}}(s)\right)}{s} d s, t \in[a, b] \text { and } n=0,1, \ldots
\end{array}\right.
$$

We now split the proof in three parts.

Step 1. Sequences $\left\{y_{n}\right\}$ and $\left\{z_{n}\right\}$ satisfy the following relation:

$$
\underline{x}(t)=y_{0}(t) \leqslant y_{1}(t) \leqslant y_{2}(t) \leqslant \ldots \leqslant y_{n}(t) \leqslant \ldots \leqslant z_{n}(t) \leqslant \ldots \leqslant z_{1}(t) \leqslant z_{0}(t)=\bar{x}(t)
$$

for $t \in[0, T]$.

First, we shall prove that sequence $\left\{y_{n}\right\}$ is nondecreasing and

$$
y_{n}(t) \leqslant z_{0}(t), t \in[a, b] \text { for all } n \in \mathbb{N} \text {. }
$$

According to the assumptions, we can know $\underline{x}(t)=y_{0}(t) \leqslant \bar{x}(t)=z_{0}(t)$ for $t \in[a, b]$ and

$$
y_{1}(t)=x_{a}+\frac{1}{\Gamma(\alpha)} \int_{a}^{t}\left(\ln \frac{t}{s}\right)^{\alpha-1} \frac{f\left(s, y_{0}(s), I_{a^{+}}^{\alpha} y_{0}(s)\right)}{s} d s \geqslant y_{0}(t) \text { for } t \in[a, b] .
$$

Since $(x, y) \mapsto f(t, x, y)$ is nondecreasing, then it is obvious that

$$
f\left(s, y_{0}(s), I_{a^{+}}^{\alpha} y_{0}(s)\right) \leqslant f\left(s, z_{0}(s), I_{a^{+}}^{\alpha} z_{0}(s)\right)
$$

for $s \in[0, T]$. This deduces

$$
y_{1}(t)=x_{a}+\frac{1}{\Gamma(\alpha)} \int_{a}^{t}\left(\ln \frac{t}{s}\right)^{\alpha-1} \frac{f\left(s, y_{0}(s), I_{a^{+}}^{\alpha} y_{0}(s)\right)}{s} d s
$$




$$
\leqslant x_{a}+\frac{1}{\Gamma(\alpha)} \int_{a}^{t}\left(\ln \frac{t}{s}\right)^{\alpha-1} \frac{f\left(s, z_{0}(s), I_{a^{+}}^{\alpha} z_{0}(s)\right)}{s} d s \leqslant z_{0}(t) \text { for } t \in[a, b] .
$$

Therefore, we assume inductively

$$
y_{n-1}(t) \leqslant y_{n}(t) \leqslant z_{0}(t) \text { for } t \in[a, b] .
$$

By virtue of definition of $\left\{y_{n}\right\}$, we have

$$
\begin{aligned}
y_{n}(t) & =x_{a}+\frac{1}{\Gamma(\alpha)} \int_{a}^{t}\left(\ln \frac{t}{s}\right)^{\alpha-1} \frac{f\left(s, y_{n-1}(s), I_{a^{+}}^{\alpha} y_{n-1}(s)\right)}{s} d s, \\
y_{n+1}(t) & =x_{a}+\frac{1}{\Gamma(\alpha)} \int_{a}^{t}\left(\ln \frac{t}{s}\right)^{\alpha-1} \frac{f\left(s, y_{n}(s), I_{a^{+}}^{\alpha} y_{n}(s)\right)}{s} d s
\end{aligned}
$$

for $t \in[0, T]$. Using the monotonicity of $f$, we readily obtain

$$
y_{n}(t) \leqslant y_{n+1}(t) \leqslant z_{0}(t) \text { for } t \in[a, b] .
$$

Further, we will show that

$$
y_{n}(t) \leqslant z_{n}(t) \text { for } t \in[a, b] \text { and } n \in \mathbb{N} \text {. }
$$

For $n=0$, it is clear that $\underline{x}(t)=y_{0}(t) \leqslant z_{0}(t)=\bar{x}(t)$ for $t \in[a, b]$. Now, we also suppose inductively

$$
y_{n}(t) \leqslant z_{n}(t), t \in[a, b] \text {. }
$$

Analogically, we easily conclude from the monotonicity of $f$ with respect to the second and the third variables that

$$
y_{n+1}(t) \leqslant z_{n+1}(t), t \in[a, b] .
$$

Also, we have that the sequence $\left\{z_{n}\right\}$ is nonincreasing.

Step 2. Sequences $\left\{y_{n}\right\}$ and $\left\{z_{n}\right\}$ are both relatively compact in $C(a, b ; \mathbb{R})$.

Because $f$ is continuous and $\underline{x}, \bar{x} \in C(a, b ; \mathbb{R})$, from Step 1 , we have $\left\{y_{n}\right\}$ and $\left\{z_{n}\right\}$ belong to $C(a, b ; \mathbb{R})$ as well. It follows from (3.2) that $\left\{y_{n}\right\}$ and $\left\{z_{n}\right\}$ are uniformly bounded. On the other hand, for any $t_{1}, t_{2} \in[a, b]$, without loss of generality, let $t_{1} \leqslant t_{2}$, we have

$$
\begin{aligned}
\left|y_{n+1}\left(t_{1}\right)-y_{n+1}\left(t_{2}\right)\right|= & \frac{1}{\Gamma(\alpha)} \mid \int_{a}^{t_{2}}\left(\ln \frac{t_{2}}{s}\right)^{\alpha-1} \frac{f\left(s, y_{n}(s), I_{a^{+}}^{\alpha} y_{n}(s)\right)}{s} d s- \\
& -\int_{a}^{t_{1}}\left(\ln \frac{t_{1}}{s}\right)^{\alpha-1} \frac{f\left(s, y_{n}(s), I_{a^{+}}^{\alpha} y_{n}(s)\right)}{s} d s \mid \\
= & \frac{1}{\Gamma(\alpha)} \mid \int_{a}^{t_{1}}\left[\left(\ln \frac{t_{2}}{s}\right)^{\alpha-1}-\left(\ln \frac{t_{1}}{s}\right)^{\alpha-1}\right] \frac{f\left(s, y_{n}(s), I_{a^{+}}^{\alpha} y_{n}(s)\right)}{s} d s \\
& +\int_{t_{1}}^{t_{2}}\left(\ln \frac{t_{2}}{s}\right)^{\alpha-1} \frac{f\left(s, y_{n}(s), I_{a^{+}}^{\alpha} y_{n}(s)\right)}{s} d s \mid \\
\leqslant & \frac{M}{\Gamma(\alpha)}\left|\int_{a}^{t_{1}} \frac{1}{s}\left[\left(\ln \frac{t_{2}}{s}\right)^{\alpha-1}-\left(\ln \frac{t_{1}}{s}\right)^{\alpha-1}\right] d s+\int_{t_{1}}^{t_{2}} \frac{1}{s}\left(\ln \frac{t_{2}}{s}\right)^{\alpha-1} d s\right| \\
\leqslant & \frac{M}{\Gamma(1+\alpha)}\left[\left(\ln \frac{t_{2}}{a}\right)^{\alpha}-\left(\ln \frac{t_{1}}{a}\right)^{\alpha}+2\left(\ln \frac{t_{2}}{t_{1}}\right)^{\alpha}\right] \\
\rightarrow & 0, \text { as }\left|t_{1}-t_{2}\right| \rightarrow 0,
\end{aligned}
$$

where $M>0$ is a constant independent of $n, t_{1}$, and $t_{2}$. It implies that $\left\{y_{n}\right\}$ is equicontinuous in $C(a, b ; \mathbb{R})$. From Arzelà-Ascoli Theorem (see [27]), we imply that $\left\{y_{n}\right\}$ is relatively compact in $C(a, b ; \mathbb{R})$. Similarly, we obtain $\left\{z_{n}\right\}$ is also relatively compact in $C([a, b] ; \mathbb{R})$. 
Step 3. There exist maximal and minimal solutions in $U_{(\underline{x}, \bar{x})}$.

Step 1 and Step 2 indicate that the sequences $\left\{y_{n}\right\}$ and $\left\{z_{n}\right\}$ are both monotone and relatively compact in $C(a, b ; \mathbb{R})$. Obviously, there exist continuous functions $y$ and $z$ with $y_{n}(t) \leqslant y(t) \leqslant z(t) \leqslant z_{n}(t)$ for all $t \in[a, b]$ and $n \in \mathbb{N}$, such that $\left\{y_{n}\right\}$ and $\left\{z_{n}\right\}$ converge uniformly to $y$ and $z$ in $C(a, b ; \mathbb{R})$, respectively. So, $y$ and $z$ are two solutions of (3.1), i.e.,

$$
\begin{aligned}
& y(t)=x_{a}+\frac{1}{\Gamma(\alpha)} \int_{a}^{t}\left(\ln \frac{t}{s}\right)^{\alpha-1} \frac{f\left(s, y(s), I_{a^{+}}^{\alpha} y(s)\right)}{s} d s, \\
& z(t)=x_{a}+\frac{1}{\Gamma(\alpha)} \int_{a}^{t}\left(\ln \frac{t}{s}\right)^{\alpha-1} \frac{f\left(s, z(s), I_{a^{+}}^{\alpha} z(s)\right)}{s} d s
\end{aligned}
$$

for $t \in[a, b]$. However, the fact (3.2) ensures that

$$
\underline{x}(t) \leqslant y(t) \leqslant z(t) \leqslant \bar{x}(t) \text { for } t \in[a, b] .
$$

Finally, we shall illustrate that $y$ and $z$ are the minimal and maximal solutions in $U_{(\underline{x}, \bar{x})}$, respectively. For any $x \in \mathrm{U}_{(\underline{x}, \bar{x})}$, then we have

$$
\underline{x}(t) \leqslant x(t) \leqslant \bar{x}(t) \text { for } t \in[a, b] .
$$

Recall that $f$ is nondecreasing with respect to the second and the third arguments, we induct

$$
\underline{x}(t) \leqslant y_{n}(t) \leqslant x(t) \leqslant z_{n}(t) \leqslant \bar{x}(t) \text { for } t \in[a, b] \text { and } n \in \mathbb{N} .
$$

Taking limits as $n \rightarrow \infty$ into the above inequality, one implies

$$
\underline{x}(t) \leqslant y(t) \leqslant x(t) \leqslant z(t) \leqslant \bar{x}(t) \text { for } t \in[a, b] .
$$

This means that $x_{L}=y$ and $x_{M}=z$ are the minimal and maximal solutions in $U_{(\underline{x}, \bar{x})}$, respectively, which completes the proof of the theorem.

Theorem 3.4. Assume that hypotheses of Theorem 3.3 are satisfied. Then fractional nonlinear differential equation (1.1) has at least one solution in $\mathrm{C}([\mathrm{a}, \mathrm{b}] ; \mathbb{R})$.

Proof. By the assumptions and Theorem 3.3, we have $\mathrm{U}_{(\underline{x}, \bar{x})} \neq \emptyset$, i.e., the solution set of fractional integral equation (3.1) is nonempty in $C(a, b ; \mathbb{R})$. This combines with Theorem 3.1 to verify that fractional nonlinear differential equation (1.1) has at least one solution in $C(a, b ; \mathbb{R})$, which completes the proof of the theorem.

\section{Examples}

In this section, we will apply foregoing theoretical results stated in Section 3 to present two simple examples to explicate the results.

Example 4.1. Consider the following Caputo-Hadamard fractional differential equation

$$
\left\{\begin{array}{l}
{ }^{C} D_{1^{+}}^{\frac{1}{2}} \chi(t)=\frac{8}{3 \sqrt{\pi}}(\ln t)^{\frac{3}{2}}-(\ln t)^{2}+\chi(t) \text { for } t \in[1, e] \\
x(1)=0 .
\end{array}\right.
$$

Proof. Let $f(t, x(t))=\frac{8}{3 \sqrt{\pi}}(\ln t)^{\frac{3}{2}}-(\ln t)^{2}+x(t)$ for $t \in[1, e]$. From Theorem 3.1, we only prove that the following fractional integral equation has at least one solution in $C(1, e ; \mathbb{R})$

$$
x(t)=I_{1^{+}}^{\frac{1}{2}}\left(\frac{8}{3 \sqrt{\pi}}(\ln t)^{\frac{3}{2}}-(\ln t)^{2}+x(t)\right) \text { for } t \in[1, e] .
$$

Indeed, we can see that $(\underline{x}(t), \bar{x}(t))=\left(0,(\ln t)^{2}+(\ln t)^{3}\right)$ is a pair of upper and lower solutions of $(4.1)$. In 
addition, $f$ is continuous and nondecreasing with respect to the second argument. We can calculate the sequences $\left\{y_{n}\right\}$ and $\left\{z_{n}\right\}$ by

$$
\left\{\begin{array} { l } 
{ y _ { 0 } ( t ) = \underline { x } ( t ) , } \\
{ y _ { n + 1 } ( t ) = I _ { 1 ^ { + } } ^ { \frac { 1 } { 2 } } f ( t , y _ { n } ( t ) ) , n = 0 , 1 , \ldots , }
\end{array} \quad \left\{\begin{array}{l}
z_{0}(t)=\bar{x}(t), \\
z_{n+1}(t)=I_{1^{+}}^{\frac{1}{2}} f\left(t, z_{n}(t)\right), n=0,1, \ldots
\end{array}\right.\right.
$$

for $t \in[1, e]$. We are now in a position to apply Theorem 3.3 to conclude $y_{n} \rightarrow y \in C(1, e ; \mathbb{R})$ and $z_{\mathrm{n}} \rightarrow z \in \mathrm{C}(1, e ; \mathbb{R})$ as $\mathrm{n} \rightarrow \infty$. In the meantime, we can get $z(\mathrm{t})=\mathrm{y}(\mathrm{t})=(\ln \mathrm{t})^{2}$ for $\mathrm{t} \in[1, \mathrm{e}]$. The approximation of sequences $\left\{y_{n}\right\}$ and $\left\{z_{n}\right\}$ to $(\ln t)^{2}$ is shown in Fig. 1 and Table 1.
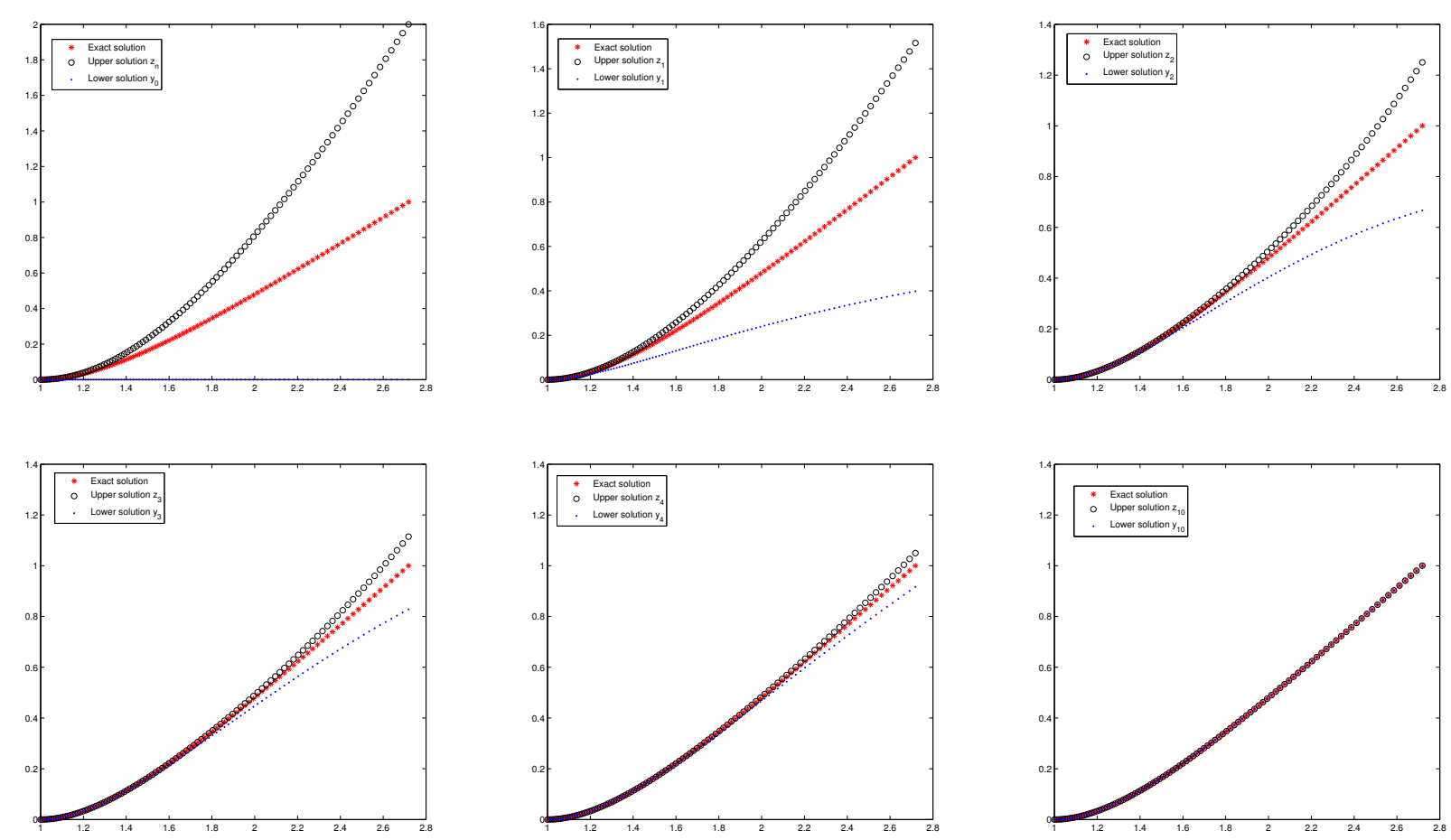

Figure 1: A plot of $y_{k}$ and $z_{k}, k=0,1,2,3,4,10$ for Example 4.1.

Table 1: Error analysis.

\begin{tabular}{|c|cccc|}
\hline & $n=5$ & $n=10$ & $n=15$ & $n=20$ \\
\hline $\sup _{t \in[1, e]}\left|y_{n}(t)-x(t)\right|$ & $8.33 \times 10^{-2}$ & $1.10 \times 10^{-3}$ & $5.51 \times 10^{-6}$ & $1.46 \times 10^{-8}$ \\
$\sup _{t \in[1, e]}\left|z_{n}(t)-x(t)\right|$ & $5.00 \times 10^{-2}$ & $1.49 \times 10^{-4}$ & $1.65 \times 10^{-6}$ & $3.51 \times 10^{-9}$ \\
\hline
\end{tabular}

Example 4.2. Consider the following Caputo-Hadamard fractional differential equation

$$
\left\{\begin{array}{l}
{ }^{C} D_{1^{+}}^{\frac{1}{2}} x(t)=\frac{2}{\sqrt{\pi}}(\ln t)^{\frac{1}{2}}-\frac{4}{3 \sqrt{\pi}}(\ln t)^{\frac{3}{2}}+I_{1^{+}}^{\frac{1}{2}} x(t) \text { for } t \in[1, e] \\
x(1)=0
\end{array}\right.
$$

Proof. Let $f\left(t, x(t), I_{1^{+}}^{\frac{1}{2}} x(t)\right)=\frac{2}{\sqrt{\pi}}(\ln t)^{\frac{1}{2}}-\frac{4}{3 \sqrt{\pi}}(\ln t)^{\frac{3}{2}}+\mathrm{I}_{1^{+}}^{\frac{1}{2}} x(t)$ for $t \in[1, e]$. Then, the corresponding fractional integral equation is obtained by

$$
x(t)=I_{1^{+}}^{\frac{1}{2}}\left(\frac{2}{\sqrt{\pi}}(\ln t)^{\frac{1}{2}}-\frac{4}{3 \sqrt{\pi}}(\ln t)^{\frac{3}{2}}+I_{1^{+}}^{\frac{1}{2}} x(t)\right) \text { for } t \in[1, e] .
$$

Obviously, $(\underline{x}(t), \bar{x}(t))=\left(0,(\ln t)^{2}+\ln t\right)$ is a pair of upper and lower solutions of (4.2). All conditions in 
Theorem 3.3 are verified readily, so, we construct the sequences $\left\{y_{n}\right\}$ and $\left\{z_{n}\right\}$ by

$$
\left\{\begin{array} { l } 
{ y _ { 0 } ( t ) = \underline { x } ( t ) , } \\
{ y _ { n + 1 } ( t ) = I _ { 1 ^ { + } } ^ { \frac { 1 } { 2 } } f ( t , y _ { n } ( t ) , I _ { 1 ^ { + } } ^ { \frac { 1 } { 2 } } y _ { n } ( t ) ) , }
\end{array} \quad \left\{\begin{array}{l}
z_{0}(t)=\bar{x}(t), \\
z_{n+1}(t)=I_{1^{+}}^{\frac{1}{2}} f\left(t, z_{n}(t), I_{1^{+}}^{\frac{1}{2}} z_{n}(t)\right) .
\end{array}\right.\right.
$$

Applying Theorem 3.3 again, we have $y_{n} \rightarrow y \in C([1, e] ; \mathbb{R})$ and $z_{n} \rightarrow z \in C(1, e ; \mathbb{R})$ as $n \rightarrow \infty$. Besides, we have that $z(t)=y(t)=\ln t$ for $t \in[1, e]$. Furthermore, we also obtain the approximation results, Fig. 2 and Table 2.
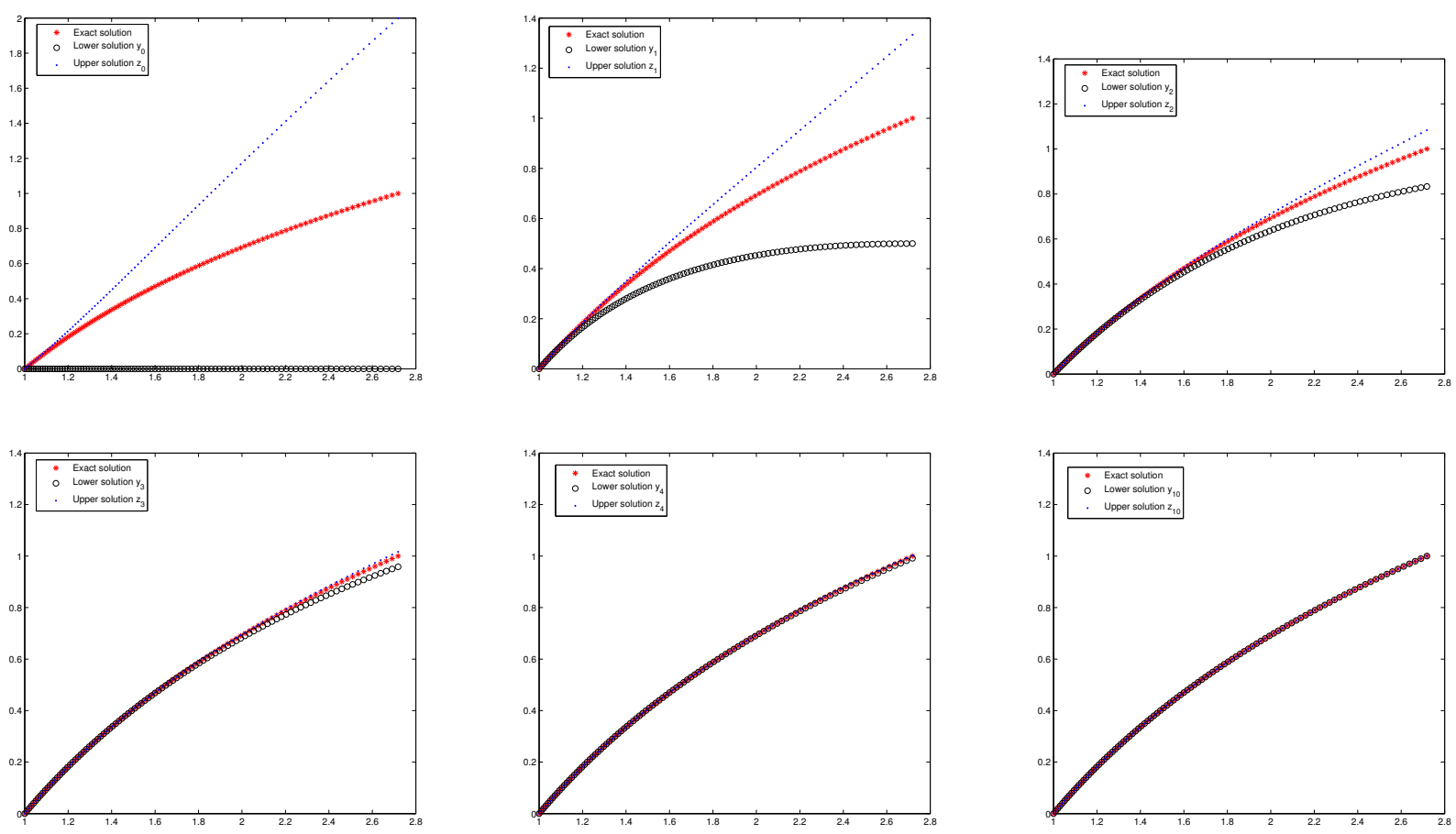

Figure 2: A plot of $y_{k}$ and $z_{k}, k=0,1,2,3,4,10$ for Example 4.2.

Table 2: Error analysis.

\begin{tabular}{|c|cccc|}
\hline Iterations & $\mathrm{n}=5$ & $\mathrm{n}=10$ & $\mathrm{n}=15$ & $\mathrm{n}=20$ \\
\hline Absolute Error & $8.34 \times 10^{-3}$ & $2.6 \times 10^{-7}$ & $7.65 \times 10^{-13}$ & $1.11 \times 10^{-16}$ \\
$\sup _{\mathrm{t} \in[1, \mathrm{e}]}\left|\mathrm{y}_{\mathrm{n}}(\mathrm{t})-x(\mathrm{t})\right|$ & $2.78 \times 10^{-3}$ & $5.01 \times 10^{-8}$ & $9.57 \times 10^{-14}$ & $1.14 \times 10^{-16}$ \\
$\sup _{\mathrm{t} \in[1, \mathrm{e}]}\left|z_{\mathrm{n}}(\mathrm{t})-x(\mathrm{t})\right|$ & & & & \\
\hline
\end{tabular}

\section{Acknowledgment}

This work was financially supported by the open fund of Key Laboratory of Numerical Simulation of Sichuan Province (Grant No. 2017ZZ001), and the Innovative Team Program of the Neijiang Normal University (Grant No. 13TD02).

\section{References}

[1] D. Baleanu, K. Diethelm, E. Scalas, J. J. Trujillo, Fractional calculus, Models and numerical methods, Series on Complexity, Nonlinearity and Chaos, World Scientific Publishing Co. Pte. Ltd., Hackensack, NJ, (2012). 1

[2] P. L. Butzer, A. A. Kilbas, J. J. Trujillo, Fractional calculus in the Mellin setting and Hadamard-type fractional integrals, J. Math. Anal. Appl., 269 (2002), 1-27.1 
[3] W. Chen, H.-G. Sun, X.-D. Zhang, D. Korošak, Anomalous diffusion modeling by fractal and fractional derivatives, Comput. Math. Appl., 59 (2010), 1754-1758. 1

[4] F.-L. Chen, Y. Zhou, Attractivity of fractional functional differential equations, Comput. Math. Appl., 62 (2011), 13591369. 1

[5] R. Chen, S.-M. Zhou, Well-posedness and persistence properties for two-component higher order Camassa-Holm systems with fractional inertia operator, Nonlinear Anal. Real World Appl., 33 (2017), 121-138. 1

[6] A. Fiscella, P. Pucci, p-fractional Kirchhoff equations involving critical nonlinearities, Nonlinear Anal. Real World Appl., 35 (2017), 350-378. 1

[7] Y. Y. Gambo, F. Jarad, D. Baleanu, T. Abdeljawad, On Caputo modification of the Hadamard fractional derivatives, Adv. Difference Equ., 2014 (2014), 12 pages. 1, 2.1, 2.2, 2, 2.3, 2.6

[8] R. Hilfer (Ed.), Applications of fractional calculus in physics, World Scientific Publishing Co., Inc., River Edge, NJ, (2000). 1

[9] F. Jarad, T. Abdeljawad, D. Baleanu, Caputo-type modification of the Hadamard fractional derivatives, Adv. Difference Equ., 2012 (2012), 8 pages. 1

[10] A. A. Kilbas, H. M. Srivastava, J. J. Trujillo, Theory and applications of fractional differential equations, North-Holland Mathematics Studies, Elsevier Science B.V., Amsterdam, (2006). 1, 2.1, 2.2, 2, 2.3, 2.6

[11] A. Kubica, P. Rybka, K. Ryszewska, Weak solutions of fractional differential equations in non cylindrical domains, Nonlinear Anal. Real World Appl., 36 (2017), 154-182. 1

[12] C.-P. Li, F.-H. Zeng, Numerical methods for fractional calculus, Chapman \& Hall/CRC Numerical Analysis and Scientific Computing, CRC Press, Boca Raton, FL, (2015).

[13] Y. Luchko, Boundary value problems for the generalized time-fractional diffusion equation of distributed order, Fract. Calc. Appl. Anal., 12 (2009), 409-422. 1

[14] R. L. Magin, Fractional calculus in bioengineering, Begell House, Redding, (2006). 1

[15] F. Mainardi, Fractional calculus and waves in linear viscoelasticity, An introduction to mathematical models, Imperial College Press, London, (2010). 1

[16] N. Pan, B.-L. Zhang, J. Cao, Degenerate Kirchhoff-type diffusion problems involving the fractional p-Laplacian, Nonlinear Anal. Real World Appl., 37 (2017), 56-70. 1

[17] I. Podlubny, Fractional differential equations, An introduction to fractional derivatives, fractional differential equations, to methods of their solution and some of their applications, Mathematics in Science and Engineering, Academic Press, Inc., San Diego, CA, (1999). 1

[18] S. G. Samko, A. A. Kilbas, O. I. Marichev, Fractional integrals and derivatives, Theory and applications, Edited and with a foreword by S. M. Nikol'skiǐ, Translated from the 1987 Russian original, Revised by the authors, Gordon and Breach Science Publishers, Yverdon, (1993). 1

[19] H.-G. Sun, W. Chen, C.-P. Li, Y.-Q. Chen, Finite difference schemes for variable-order time fractional diffusion equation, Internat. J. Bifur. Chaos Appl. Sci. Engrg., 22 (2012), 16 pages. 1

[20] H.-G. Sun, W. Chen, H. Wei, Y.-Q. Chen, A comparative study of constant-order and variable-order fractional models in characterizing memory property of systems, Eur. Phys. J., 193 (2011), 185-192. 1

[21] F. Tao, X. Wu, Existence and multiplicity of positive solutions for fractional Schrödinger equations with critical growth, Nonlinear Anal. Real World Appl., 35 (2017), 158-174. 1

[22] G.-C. Wu, D. Baleanu, Z.-G. Deng, S.-D. Zeng, Lattice fractional diffusion equation in terms of a Riesz-Caputo difference, Phys. A, 438 (2015), 335-339. 1

[23] G.-C. Wu, D. Baleanu, S.-D. Zeng, Discrete chaos in fractional sine and standard maps, Phys. Lett. A, 378 (2014), 484-487. 1

[24] X.-J. Yang, A new integral transform operator for solving the heat-diffusion problem, Appl. Math. Lett., 64 (2017), 193197. 1

[25] X.-J. Yang, D. Baleanu, H. M. Srivastava, Local fractional integral transforms and their applications, Elsevier/Academic Press, Amsterdam, (2016).

[26] X.-J. Yang, J. A. T. Machado, A new fractional operator of variable order: application in the description of anomalous diffusion, Phys. A, 418 (2017), 276-283. 1

[27] E. Zeidler, Nonlinear functional analysis and its applications, II/B, Nonlinear monotone operators, Translated from the German by the author and Leo F. Boron, Springer-Verlag, New York, (1990). 3

[28] S.-D. Zeng, D. Baleanu, Y.-R. Bai, G.-C. Wu, Fractional differential equations of Caputo-Katugampola type and numerical solutions, Appl. Math. Comput., 315 (2017), 549-554. 1

[29] F.-H. Zeng, C.-P. Li, F.-W. Liu, I. Turner, The use of finite difference/element approaches for solving the time-fractional subdiffusion equation, SIAM J. Sci. Comput., 35 (2013), 2976-3000.

[30] Y.-N. Zhang, Z.-Z. Sun, X. Zhao, Compact alternating direction implicit scheme for the two-dimensional fractional diffusion-wave equation, SIAM J. Numer. Anal., 50 (2012), 1535-1555.

[31] X. Zhao, Z.-Z. Sun, A box-type scheme for fractional sub-diffusion equation with Neumann boundary conditions, J. Comput. Phys., 230 (2011), 6061-6074. 1

[32] P. Zhuang, F. Liu, V. Anh, I. Turner, Numerical methods for the variable-order fractional advection-diffusion equation with a nonlinear source term, SIAM J. Numer. Anal., 47 (2009), 1760-1781. 1 